\title{
Time Synchronization in Wireless Sensor Network based on Level Discovery Phase
}

\author{
Deeksha Verma \\ Dept. of computer Applications \\ Kanpur Institute of Technology, Kanpur \\ India
}

\author{
Arun Kumar Tripathi \\ Associate Professor \\ Krishna Institute of Engineering and \\ Technology, Ghaziabad, India
}

\begin{abstract}
Time Synchronization aims towards synchronizing the local time for some or all nodes in the network is a necessity for many applications. Time synchronization help to find the computer clock difference and real time difference of transmission of message in wireless sensor network. We proposed a new technique based on time synchronization in wireless sensor network. This technique is derived by message exchange of level discovery phase in time synchronization protocol. We explain the basic level discovery phase technique to understand the time of data transmission in between sensor nodes and to make improvement in new and large area of sensor network.
\end{abstract}

\section{Keywords}

WSN, intruder, pulse delay attack, time synchronization, computer clock difference, real time difference, angle-of arrival.

\section{INTRODUCTION}

A Wireless Sensor Network (WSN) [1] is the network of sensor nodes that can sense the environment and communicate the information in wireless medium. WSN is the collection of sensor nodes with limited resources that collaborate in order to achieve a common goal. WSN is the distributed and self organized network. WSN is the viable solutions for a wide variety of real-world challenges. WSN must track the threats and attacks from intruder [2] because these areas are sometimes physically accessible to intruder who would like to acquire the physical location of sensor node by providing wrong command to the sensor network [3].

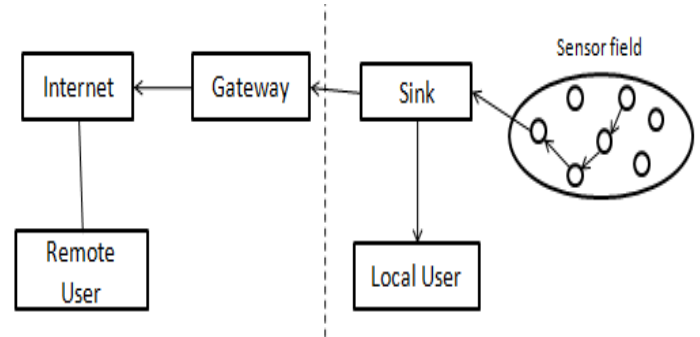

Fig.1: Wireless Sensor Network

Wireless Sensor Network can be stationary or mobile. In stationary wireless sensor network (SWSN), the sensor nodes are stationary or static, which means the sensor nodes are deployed randomly and after deployment the position of nodes do not change. In Mobile wireless sensor network (MWSN) sensor nodes can move, and after deployment they can interact with the physical environment by controlling own movement, and can appear at different location at different time. WSNs are composed of embedded systems that are capable of:

* Interact with environment through various sensor nodes.

* Processing information.

* Communicate information wirelessly in the network or with their neighbors.

Wireless sensor network can capture only one or few replicated nodes those having the same identity with the captured node and finally deploy number of replica throughout the network. Node replication attack harms the wireless sensor network by many ways, these are given below:

* It harm to the network because the replicated node also have the same identity as the sensor node.

* It creates various attacks by extracting all the secrets from sensor nodes.

* It corrupts the operation by providing false information.

* It can stop the processing of network.

* Difficult to detect replicated node and hence authentication is difficult.

\section{RELATED WORK}

In present's time, many protocols are proposed by researchers for Secure Pair Wise Synchronization (SPS) [4] protocol for sender-receiver $[5,6]$ based protocol and node replication attack. Secure pair wise synchronization (SPS) protocol is considered as sender receiver based protocol [7].

In sender-receiver based protocol, sender gets synchronized with receiver, and if they get synchronized they start communication with each other and check the time delay and clock difference between two nodes of sending and receiving response. The message delay [6] between sender and receiver is measure the total time taken, from receiver request to receiving a response to receiver.

\section{PROBLEM IDENTIFIED}

Researchers have proposed number of protocols for time synchronization. These protocols are associated with security in Wireless Sensor Network. Some protocols are suitable for the message delay and some protocols are suitable for the computer clock difference and real time difference, but not a single solution can find the message delay as well as computer clock difference and real time difference in wireless sensor network.

Time synchronization protocol in wireless sensor network [8] is used to detect pulse delay attack. In this paper, the pulse attack through a comparison of the message delay $d$, with maximum speed message delay $d^{*}$. If the calculate delay is greater than the 
maximum delay, it means there is any replicated node in the sensor network who is interrupting the transmission between nodes in WSN.

In Detection and mitigation of node replication attack in WSN [9] is used to detect both pulse delay attack and node replication. This paper is only finding the message delay in the sensor network but it is not able to find the computer clock difference and real-time difference of message transmission in between two sensor node of wireless sensor network.

In [10], the sensor nodes are aware about their physical position in wireless sensor network. This paper introduces a localization technique based on angle-of -arrival between sensor nodes in a particular area of wireless sensor network. Fig.2. represent the basic localization technique to understand the angle-of-arrival of sensor nodes and to make progress in a new and large open area of sensor network localization.

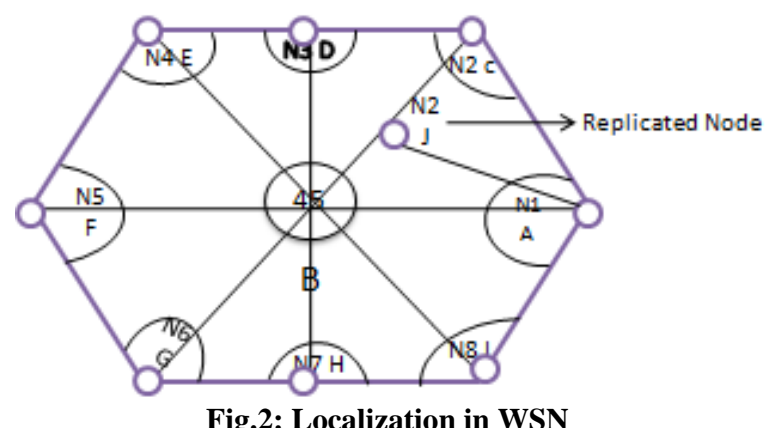

\section{PROPOSED WORK}

Many techniques are used to protect the wireless sensor network from replicated node, these techniques are aware about their time of arrival in wireless sensor network. In any wireless sensor network, as the time increases the input power supply to the node is decreases which cause change in frequency of the operation. Due to this effect different nodes have different frequency of operation from time to time; by this approach local time varies from node to node.

By the above factor we can understand that the each node having different local time, but how far the local clock time difference can affect the operation of any process is an important question. To answer this question, we use the time synchronization protocol for the sensor nodes. This synchronization contains two phase: a level discovery phase and a synchronization phase [11, 12][13-15].

The level discovery phase is the process of building a network topology, and each node is assigned in a level. The arrangement of node in level form is represented in below figure.

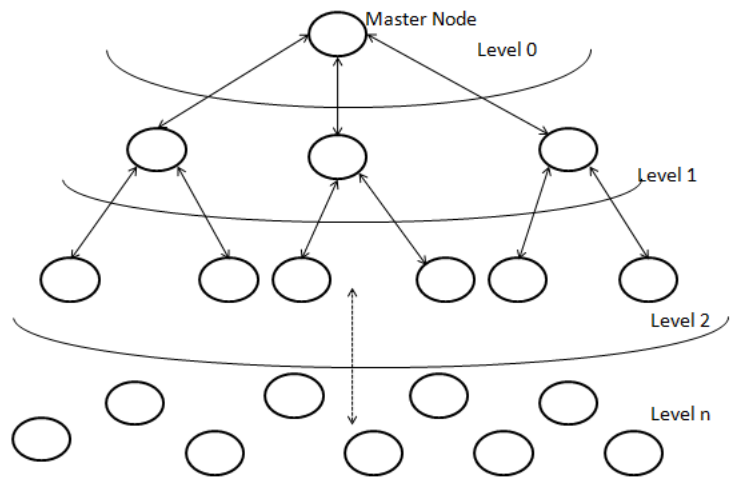

Fig 3: Level discovery phase in time synchronization

In the above figure, the first step is to select the root node as the master node. The master node can be connected to sensor node as its reference, such as GPS, it is assigned to level 0 . Then the root node broadcast the level discovery packets, which contains their own level, identities and all the neighboring nodes, it is assigned as level 1. Nodes, which have received these level discovery packets, continue to send packets, which have their own level information. By this all nodes in the network are assigned in a level.

The synchronization phase is the process of synchronizing all of sensor nodes in the network. In the synchronization phase all the nodes in the network have been assigned a level, the root node send the time synchronization packets. Node of level $n$ synchronizes to a node of level $n$-1through the two way communication. The node of level $\mathrm{n}$ sends the packet with its local sent timeT1. The node of level n-1 receives the packets with its local time $\mathrm{T} 2$, which can be calculated by following equation,

Where $\mathrm{d}$ is the propagation delay

And $\Delta$ is the relative clock difference between two nodes [16, 17]

$$
\mathrm{T} 2=\mathrm{T} 1+\mathrm{d}+\Delta
$$

Then, after a random time, node of level n-1 respond back to the node of level $n$ through an acknowledgement packet of time T3, which include the value of T1, T2, T3 and its level information. Node of level $\mathrm{n}$ will receive this packet at time T4, which represent in figure. 2 and it help to calculate the value of $d$ and $\Delta$, which can be calculated by below equation and synchronizes itself to node of level n-1. At the end of synchronization phase, all the node in the network became synchronized.

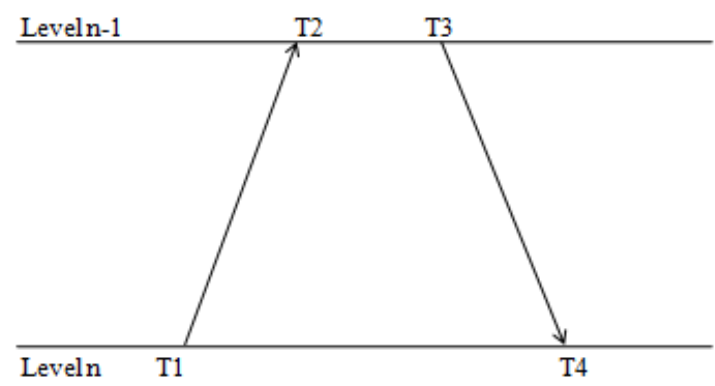

Fig 4: Message exchange of level discovery phase in time synchronization protocol. 


$$
\begin{aligned}
& \Delta=(\mathrm{T} 2-\mathrm{T} 1)-(\mathrm{T} 4-\mathrm{T} 3) / 2 \\
& \mathrm{~d}=(\mathrm{T} 2-\mathrm{T} 1)+(\mathrm{T} 4-\mathrm{T} 3) / 2
\end{aligned}
$$

By the above two equation we can calculate the propagation delay $\mathrm{d}$ and relative clock difference between two nodes $\Delta$.

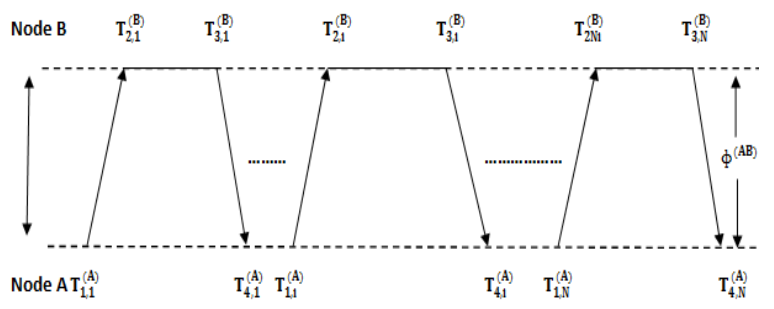

Fig 5: Clock Synchronization for SRS

Timing-sync protocol for sender-receiver synchronization [20] that exploits the two way message exchange mechanism to achieve the synchronization between two nodes in figure (5). Figure (5) represents $\mathrm{N}$ two way message exchanges between two nodes, node $\mathrm{A}$ and node $\mathrm{B}$, which are assumed two exhibit the clock phase offset difference $\phi^{(\mathrm{AB})}$. The time stamps $\mathrm{T}_{1, \mathrm{i}}^{(\mathrm{A})}, \mathrm{T}_{4, \mathrm{i}}^{(\mathrm{A})}, \mathrm{T}_{2, \mathrm{i}}^{(\mathrm{B})}, \mathrm{T}_{3, \mathrm{i}}^{(\mathrm{B})}$ are made during the $\mathrm{i}^{\text {th }}$ message exchange measured by the local clocks of node $\mathrm{A}$ and node $\mathrm{B}$. The time stamp for $\mathrm{T}_{2, \mathrm{i}}^{(\mathrm{B})}, \mathrm{T}_{4, \mathrm{i}}^{(\mathrm{A})}$ can be expressed given below:

$$
\begin{aligned}
& \mathrm{T}_{2, \mathrm{i}}^{(\mathrm{B})}=\mathrm{T}_{1, \mathrm{i}}^{(\mathrm{A})}+\phi^{(\mathrm{AB})}+\mathrm{d}^{(\mathrm{AB})}+\mathrm{Xi}^{(\mathrm{AB})} \\
& \mathrm{T}_{4, \mathrm{i}}^{(\mathrm{A})}=\mathrm{T}_{3, \mathrm{i}}^{(\mathrm{B})}+\phi^{(\mathrm{BA})}+\mathrm{d}^{(\mathrm{BA})}+\mathrm{Xi}^{(\mathrm{BA})}
\end{aligned}
$$

Where $\mathrm{d}^{(\mathrm{AB})}$ and $\mathrm{Xi}^{(\mathrm{AB})}$ are the fixed and random portion of timing delay in message transmission between nodes $\mathrm{A}$ and $\mathrm{B}$ respectively and time differences are defined as:

$$
\begin{aligned}
\mathrm{Ui} \triangleq \mathrm{T}_{2, i}^{(\mathrm{B})}-\mathrm{T}_{1, i}^{(\mathrm{A})} \text { and } \\
\mathrm{Vi} \triangleq \mathrm{T}_{4, i}^{(\mathrm{A})}-\mathrm{T}_{3, i}^{(\mathrm{B})}
\end{aligned}
$$

Where $\mathrm{Ui}$ and $\mathrm{Vi}$ are the average delay observation in one round of message exchange. Two nodes A and B exchange only one time of message exchange $(\mathrm{N}=1)$, without statistical model for the variable delay components $\mathrm{X}_{1}^{(\mathrm{AB})}$ and $\mathrm{X}_{1}^{(\mathrm{BA})}$ in equation (4) and (5). The simple estimate for $\phi^{(\mathrm{AB})}$ is proposed as:

$$
\phi^{(\mathrm{AB})}=\frac{\mathrm{U} 1-\mathrm{V} 1}{2}
$$

The original form of TPSN does not estimate the clock skew; therefore frequent application of TPSN is needed to keep the clock offset between two nodes. The clock offset between two nodes keeps increasing due to the difference in the clock parameters of each round of message exchange. Therefore, applying a clock skew correction mechanism increases the synchronization accuracy and long-term reliability of synchronization.

\section{CONCLUSION AND FUTURE WORK}

This paper proposed novel method for time synchronization in wireless sensor network. This approach is more comprehensive understanding and solution space of localization and time synchronization in sensor network. It does not depend on the transmission range. It helps in improving the synchronization accuracy, reducing synchronization power consumption, and improving the scalability and robustness by determining the propagation delay and clock difference between two nodes in wireless sensor network. This is helpful to achieving message delay estimation by using acknowledgement and determines the exact timing of message transmission between nodes in wireless sensor network.

In future researchers may focus on the implementation of the sender and receiver algorithm for data transmission in wireless sensor network and also try to make it better, secure, energy efficient and cost efficient.

\section{REFERENCES}

[1] Mukherjee, B., Ghoshal, D., Yick,J, "Wireless sensor network survey," Computer Network 52(12), 2292 2330(2008).

[2] M. Zhang, V .Khsnspure, S.Chen; and X.Xiao, "Memory Efficient protocols for detecting node replication attack in Wireless Sensor Networks," in proceeding of $17^{\text {th }}$ IEEE international conference on Network Protocols (ICNP'09).

[3] Y.Zeng, J,Cao, S.Zhang, S.Gao and L.Xie, "Random Walk Based approach to detect clone attacks in Wireless Sensor Network," IEEE journal on selection are in communication VOL.28, no.5,pp. 677-691, 2010.

[4] Ganeriwal, S., Popper, C., Capkun, S., Srivastava, M.B., "Secure Time Synchronization in Sensor Networks," ACM Transaction on information and system security, Article No: 23, 11(4), 2008.

[5] Li, H., Chen, K., Wen, M., Zheng, Y, "A Secure Time Synchronization Protocol for sensor Network," Washio., Zhou, Z-H., Huang, J.Z., Hu, X., Li, J., Xie, C., He, J., Zou,D., Li,K-C., Freire, M.M(eds.) PAKDD 2007. (LNAI), Vol. 4819, pp. 515-526. Springer, Heidelberg, 2007.

[6] Song, H., Zhu, G.C.S., "Attack Resilient time synchronization for wireless Sensor Network," IEEE international Conference on Mobile Adhoc and Sensor System Conference, pp. 772, 2005.

[7] Wang, C., Ning, P., Sun, K., "Secure and resilient clock synchronization in Wireless Sensor Networks," IEEE journal on selected areas in Communications 24(2), 395408, 2006

[8] Umrao, S., Kumar, A., and Umrao, P., "Security attack and their countermeasures along with node replication attack for time synchronization in wireless sensor network" IEEE Conference INANMEET, pp. 653-658, 2013.

[9] Umrao, S., Verma, D., Tripathi, A.K., "Detection and Mitigation of node Replication with pulse delay attacks in wireless sensor network," IEEE Conference MITE, pp. 390-392, 2013.

[10] Deeksha Verma, Sachin Umrao, Rahul Verma and Arun Kumar Tripathi "A Localization Technique in Wireless Sensor network Based on angle of arrival," International journal of Computer and Applications 98(7): 26-29, July, 2014

[11] K.Nah, Q. Chaudhari, E.Serpedim and B.Suter "Analysis of clock offset and skew estimation in timing-sync protocol for sensor network," IEEE GLOBECOM 2006 proceedings, 2006. 
[12] A.B. Kulakli, K. Erciyes, "Time synchronization algorithm based on timing- sync protocol in Wireless Sensor Network," IEEE, 2008.

[13] X.Tian, X.Miao,W.Xu, B.Fan, J.Pan, "Research on time synchronization for Wireless Sensor Network based on Bayesian Estimation," 2010 Asia- Pacific Conference on Weasable Computing system, 2010.

[14] S.Xu, P.Wang, X.Huang, W.Shi, X.Xu, "Analysis of research development on time synchronization algorithm for Wireless Sensor Network," Microcontrollers and Embedded Systems, VOL.5.2011.

[15] X.Feng, H.Gao, "Research on Time Synchronization of Wireless Sensor Network," Development and Application of Computer, VOL.25,No.5,2011.
[16] Ki.Cheng, K.Lui, Y.Wu and V.Tan, "A distributed multihop time synchronization protocol for Wireless Sensor Networks using Pair Wise Broadcast Synchronization," IEEE, 2009.

[17] F.Zhang and G.Y.Deng, "Probabilistic time Synchronization in Wireless Sensor Network," IEEE2005.

[18] S.M.Lasassmeh and J.M.Conrad, "Time Synchronization in Wireless Sensor Networks: A Survey,” IEEE, 2010.

[19] S.Ganeriwal, R.Kumal and M.Srivastava, "Timing sync Protocol for Sensor Network," ACM Sensys, Los Angeles, November 2003. 